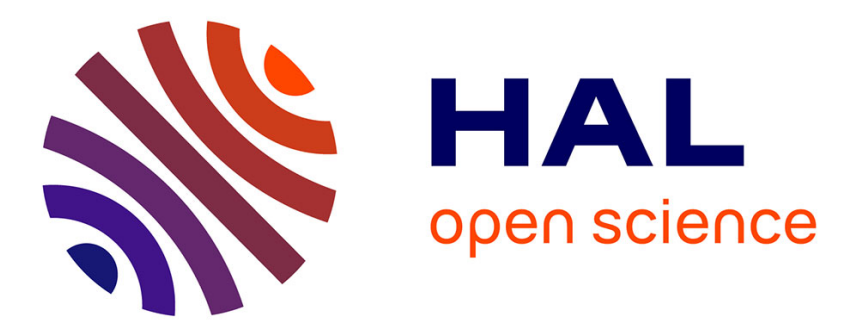

\title{
Modélisation de la propagation ultrasonore en milieu dispersif; mesure de vitesse et application à la caractérisation des graphites
}

\author{
A. Mouchtachi, Rachid El Guerjouma, J. Baboux, Y. Jayet
}

\section{To cite this version:}

A. Mouchtachi, Rachid El Guerjouma, J. Baboux, Y. Jayet. Modélisation de la propagation ultrasonore en milieu dispersif; mesure de vitesse et application à la caractérisation des graphites. Journal de Physique IV Proceedings, 1994, 04 (C5), pp.C5-909-C5-912. 10.1051/jp4:19945199 jpa-00252882

HAL Id: jpa-00252882

https://hal.science/jpa-00252882

Submitted on 1 Jan 1994

HAL is a multi-disciplinary open access archive for the deposit and dissemination of scientific research documents, whether they are published or not. The documents may come from teaching and research institutions in France or abroad, or from public or private research centers.
L'archive ouverte pluridisciplinaire HAL, est destinée au dépôt et à la diffusion de documents scientifiques de niveau recherche, publiés ou non, émanant des établissements d'enseignement et de recherche français ou étrangers, des laboratoires publics ou privés. 


\title{
Modélisation de la propagation ultrasonore en milieu dispersif ; mesure de vitesse et application à la caractérisation des graphites
}

\author{
A. MOUCHTACHI, R. EL GUERJOUMA, J.C. BABOUX et Y. JAYET
}

Laboratoire de Traitement du Signal et Ultrasons, URA 1216 du CNRS, Institut National des Sciences Appliquées de Lyon, 20 Av. A. Einstein, 69621 Villeurbanne cedex, France

\begin{abstract}
The measurement of the velocity of propagation of ultrasonic pulse in dispersive media gives a quantity which physical meaning is ambiguous. In this paper we simulate a dispersive medium using the Kramers-Krönig relationship which expresses the causality of a linear system. We develope an appropriate signal processing such as Hilbert transform and crosscorrelation to measure the time of flight of an ultrasonic impulse propagating in such a medium. This allows to deduce from this simulation the physical significance of the two informations given by two methods. Then these two methods are applied to several categories of graphites with dispersif character. Thus the differences between the measured velocities by Hilbert transform and cross-correlation are correlated to several microstructural properties of the material such as grain size, porosity and to thermal elaboration treatement.
\end{abstract}

\section{INTRODUCTION}

Les méthodes ultrasonores consistent, en général, en la mesure du temps de vol d'une impulsion se propageant sur une distance connue dans un milieu donné. Si le milieu support de la propagation est non dispersif, ce temps est relié simplement et directement à la vitesse de phase[4]. Mais dés que l'on considère un milieu dispersif, le sens physique de la vitesse mesurée devient incertain. L'objet de cette étude est d'essayer de préciser la signification physique de la vitesse mesurée en régime impulsionnel dans un milieu dispersif. Un tel milieu est modélisé à partir des relations de Kramers-Kronig [2,3] exprimant la causalité d'un système linéaire. Deux méthodes de traitement des signaux à savoir la fonction d'intercorrélation [7] et la transformée de Hilbert [1] de la réponse impulsionnelle du milieu sont utilisées pour la mesure du retard séparant la réponse du milieu à l'excitation. Nous montrons par simulation que les deux méthodes sont équivalentes pour un milieu non dispersif. En présence de dispersion elles donnent des informations différentes fonction de la dispersion. Ces deux méthodes sont ensuite appliquées à plusieurs graphites à caractère dispersif résultant de tailles de grains différentes et de taux de porosité variables. Ainsi les différences de vitesses mesurées par la transformée de Hilbert et par intercorrélation sont reliées à plusieurs phénomènes tels que la taille des grains, les taux de porosité ou encore les traitements thermiques d'élaboration modifiant la microstructure du matériau.

\section{SIMULATION}

\subsection{Modélisation d'un milieu non dispersif}

Une modélisation simple des signaux acoustiques impulsionnels réels consiste à moduler une sinusoïde par une enveloppe exponentielle [6]. L'introduction d'un filtre à retard pur de réponse impulsionnelle $h(t)=\delta(t-\tau)$ permet d'obtenir deux impulsions $x(t)$ et $y(t)$ décalé de $\tau$ à l'aide d'un produit convolution simple. 
Le calcul de la fonction d'intercorrélation de $x(t)$ et de $y(t)$ ainsi que de la transformée de Hilbert de $h(t)$ montre que le maximum de la première et le passage à zéro de la seconde correspondent exactement au décalage $\tau$ introduit entre les deux signaux.
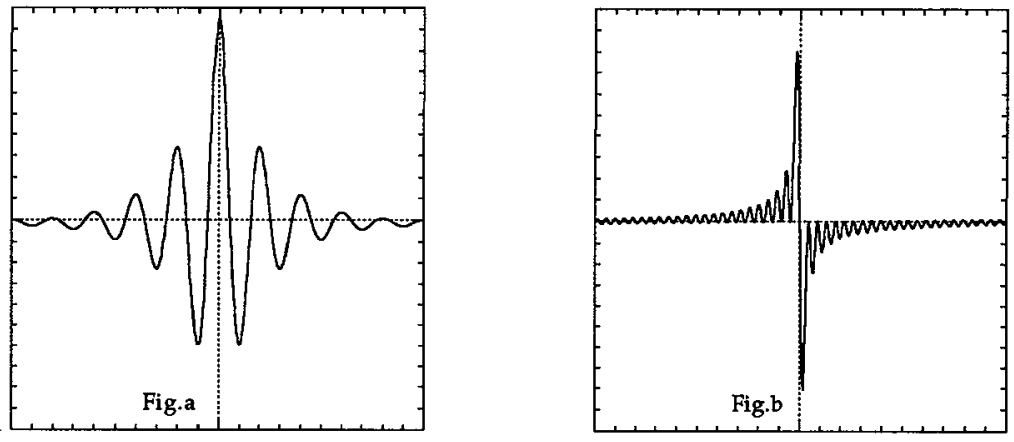

Figure1:a)intercorrélation du signal d'entrée et du signal de sortie sans dispersion. b) Transformée de Hilbert de la réponse impulsionnel $h(t)$

On montre clairement que ces deux méthodes sont équivalentes pour un milieu non dispersif.

\subsection{Modélisation d'un milieu dispersif}

La propagation dans un milieu dispersif non seulement retarde le signal de l'onde propagée mais modifie également son contenu spectral. En effet, une impulsion peut être considérée comme étant la somme infinie d'ondes monochromatiques. Chacune de ces ondes, dans un milieu dispersif va donc se propager en s'atténuant avec une vitesse de phase particulière. A une distance d donnée les différentes composantes de l'impulsion vont donc présenter entre elles des déphasages dus aux différences entre les temps de propagation. En conséquence l'impulsion voit sa forme changer au cours de la propagation et les caractéristiques du milieu à savoir l'atténuation et la vitesse de phase évoluent en fonction de la fréquence. L'expression du cœefficient d'atténuation en fonction de la fréquence est déduite à partir des relations de causalité de Kramers-Krönig[3]. Pour l'atténuation nous avons :

$$
\alpha(v)=\frac{\pi^{2} v^{2} \mathrm{dC}(v)}{C(v) \mathrm{d} v} \quad, v \text { étant la fréquence. }
$$

Pour la relation donnant la vitesse de phase $C(v)$, en fonction de la fréquence, nous la considérons comme la somme d'un terme sans dispersion et d'un terme lié directement à la dispersion.

$$
C(v)=C_{0}(1+\beta v) \text {. }
$$

Où $C_{0}$ est la vitesse de phase sans dispersion à la fréquence nulle; $\beta$ étant un cœfficient rendant compte de la dispersion. Ces deux relations permettent donc de modéliser un milieu dispersif. L'expression de la réponse impulsionnelle dans le domaine de Fourrier ou celle de la fonction de transfert d'un tel milieu est déduite des relations précédantes et s'écrit sous la forme suivante :

$$
H(v)=e^{-\alpha(v)} e^{-j k(v) d}
$$

Où $k(v)$ est le nombre d'onde, $d$ la distance de propagation.

Grâce à cette modélisation nous pouvons obtenir par simulation des signaux impulsionnels se propageant dans un milieu plus ou moins dispersif selon la valeur du paramètre $\beta$. 

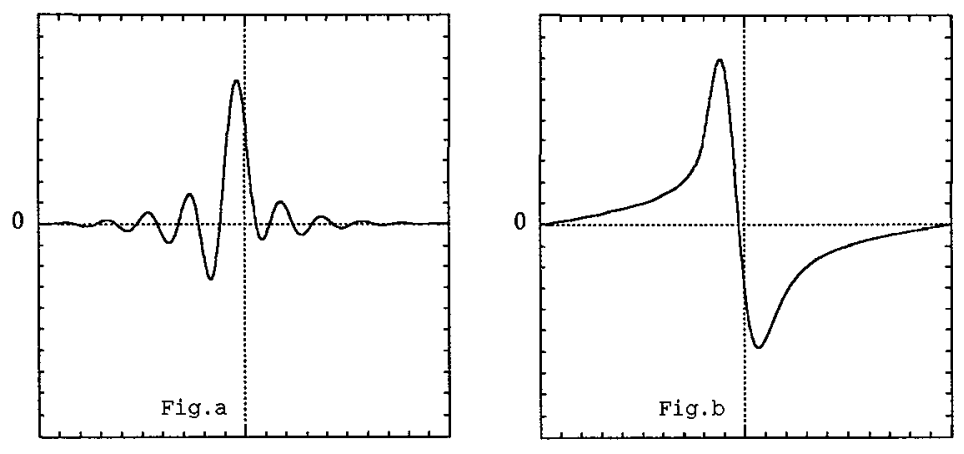

Figure2: c) Fonction d'intercorrélation du signal d'entrée et du signal de sortie dispersé. d) transformée de Hilbert de $h(t)$ en présence de dispersion.

Ainsi pour une valeur de $\beta$ assez faible, le signal de sortie subit une atténuation d'amplitude sans dispersion notable de la phase. Quand on amplifie cette dispersion la distribution fréquentielle du signal temporel change. Ainsi le maximum de la fonction d'intercorrélation, pour cette dispersion importante, (illustrée sur la figure 2a) ne coïncide plus avec le retard en absence de dispersion, de même pour le passage à zéro de la transformée de Hilbert. L'information recueillie par la transformée de Hilbert, en présence de dispersion, est altérée mais moins que le maximum de la fonction d'intercorrelation. Ces deux méthodes sont équivalentes pour des valeurs de $\beta$ faibles, par contre quand la dispersion (i.e. $\beta$ ) augmente l'écart temporel entre ces deux méthodes devient important. A titre indicatif quand nous passons d'une trés faible dispersion à une grande dispersion ( $\beta$ est multiplié par 25) l'écart des temps obtenus par transformée de Hilbert et par intercorrélation passe de lns à 63ns.

\section{APPLICATION A LA CARACTERISATION DES GRAPHITES.}

Nous disposons d'échantillons prismatiques $60 \times 60 \times 10 \mathrm{~mm}^{3}$ tirés d'un bloc cylindrique ou rectangulaire d'axe de filage $Z$. Ils sont taillés parallèlement et perpendiculairement à l'axe de filage. Les dimensions des grains sont de $0.2 \mathrm{~mm}$ (grains fins), 0.8 et $1.6 \mathrm{~mm}$ (gros grains ). Les échantillons ont des porosités variant de 10 à $30 \%$ selon qu'ils sont imprégnés ou non.

L'étude spectrale des échantillons montre que plus les dimensions des grains augmentent plus l'atténuation du milieu accompagnée d'un changement de distribution des composantes fréquentielles est forte, conséquence du phénomène de dispersion. Les mesures de vitesses par intercorrélation et par la transformée de Hilbert et les densités apparentes ou les porosités de chaque échantillon sont résumées dans les tableaux suivants :

Tableau1. grain $0,2 \mathrm{~mm}$.

\begin{tabular}{|l|l|l|l|l|}
\hline Eprouvette & $\begin{array}{l}\text { Corrélation } \\
\text { Vitesse } \\
(\mathrm{m} / \mathrm{s})\end{array}$ & $\begin{array}{l}\text { Hilbert } \\
\text { Vitesse } \\
(\mathrm{m} / \mathrm{s})\end{array}$ & $\begin{array}{l}\Delta \mathrm{V}_{\mathrm{HC}} \\
(\mathrm{m} / \mathrm{s})\end{array}$ & D.A \\
\hline F-XY & 2766 & 2769 & 3 & 1,55 \\
\hline FI-XY & 2257 & 2256 & 1 & 1,76 \\
\hline$* *$ & $* *$ & $* *$ & $* *$ & $* *$ \\
\hline F-Z & 2839 & 2838 & 1 & 1,55 \\
\hline FI-Z & 2700 & 2701 & 1 & 1,76 \\
\hline
\end{tabular}

Tableau2. grain $0,8 \mathrm{~mm}$.

\begin{tabular}{|l|l|l|l|l|}
\hline Eprouvette & $\begin{array}{l}\text { corrélation } \\
\text { vitesse }(\mathrm{m} / \mathrm{s})\end{array}$ & $\begin{array}{l}\text { Hilbert } \\
\text { vitesse }(\mathrm{m} / \mathrm{s})\end{array}$ & $\begin{array}{l}\Delta \mathrm{V}_{\mathrm{HC}} \\
(\mathrm{m} / \mathrm{s})\end{array}$ & $\begin{array}{c}\text { Porosité } \\
\%\end{array}$ \\
\hline P-XY & 1898 & 1943 & 45 & 21.56 \\
\hline PIXY & 2164 & 2196 & 32 & 20.11 \\
\hline PIIXY & 2244 & 2266 & 22 & 11.36 \\
\hline
\end{tabular}


Tableau3. grain $1,6 \mathrm{~mm}$.

\begin{tabular}{|c|c|c|c|c|}
\hline Eprouvette & $\begin{array}{l}\text { Correlation } \\
\text { Vitesse }(\mathrm{m} / \mathrm{s})\end{array}$ & $\begin{array}{c}\text { Hibert } \\
\text { Vitesse(m/s) }\end{array}$ & $\begin{array}{c}\Delta \mathrm{V}_{\mathrm{HC}} \\
(\mathrm{m} / \mathrm{s})\end{array}$ & $\begin{array}{c}\text { Porosité } \\
(\%)\end{array}$ \\
\hline AI-XY & 2190 & 1925 & 256 & 15.67 \\
\hline AI-Z & 2951 & 3065 & 114 & 15.67 \\
\hline
\end{tabular}

Les vitesses mesurées par transformée de Hilbert et par intercorrélation sont d'autant plus différentes que la taille des grains est grande et que le matériau est poreux. Ces deux méthodes sont équivalentes pour les échantillons à grains fins (tableau 1) et présentent un écart de vitesse plus important pour les échantillons non imprégnés à gros et à gains moyens. En effet, les graphites à grains fins présentant une dispersion faible, $\Delta \mathrm{V}_{\mathrm{HC}}$ ne dépasse pas $0.1 \%$, par contre pour les échantillons de tailles de grains 0.8 et $1.6 \mathrm{~mm}$ cette différence de vitesses augmente et est respectivement de $2.3 \%$ et $13 \%$. Pour un même échantillon donc une même taille de grain, $\Delta \mathrm{V}_{\mathrm{HC}}$ diminue avec les cycles d'imprégnations supplémentaires c'est à dire avec la porosité, ainsi pour l'échantillon $\mathrm{P}$ la porosité varie de 21.56 à $11.36 \%$ et $\Delta \mathrm{V}_{\mathrm{HC}}$ décroît de 2.3 à $0.97 \%$. Ces résultats confirment ceux obtenus lors de la simulation et on montrent que l'exploitation des deux méthodes permet de caractériser la dispersion c'est à dire la microstructure du matériau.

\section{CONCLUSION}

L'intérêt de cette étude était au travers d'une modélisation de la dispersion de développer un traitement de signal approprié pour la mesure du temps de vol d'une impulsion ultrasonore se propageant dans un milieu dispersif et par suite de préciser la signification physique de la vitesse de propagation qui en découle. Par simulation numérique nous avons montré que ces deux méthodes sont équivalentes pour des dispersions faibles et présentent un écart non négligeable lorsque la dispersion devient importante. La différence de vitesses mesurées par ces deux méthodes se révèlent comme un moyen intéressant de caractérisation de la microstructure telles que la porosité, la granulométrie comme cela a été montré sur des échantillons de graphites.

\section{REFERENCES BIBLIOGRAPHIQUES}

[1] Audoin B. et Roux J.Revue Phys. Appl. (25) 1011-1017 (1990)

[2] Beltzer A.I.J.Acout. Soc. Am. 73(1), Jan 1983

[3] O'Donnel M., Jaynes E.T. and Miller J.G.J. Acout. Soc. Am. 69(3), March 1983.

[4] Roux J., Hosten B., Castagnede B. et Deschanps M. Revue Phys Appl.(20) 351-358 (1985)

[5] A.Mouchtachi, R. El Guerjouma. J.C. Baboux. Journées GAPSUS Le Mans Mars 1993.

[6] R. El Guerjouma, A.Mouchtachi, Y. Jayet et J.C. Baboux. IEEE Ultrasonic Symposium octobre Tucson, U.S.A. 1993

[7] F.De. Coulon " Théorie et traitement des signaux" Dunod, 1984.

[8] J. Max."Techniques numériques du signal" Tome 1 Paris, Masson, 1977. 